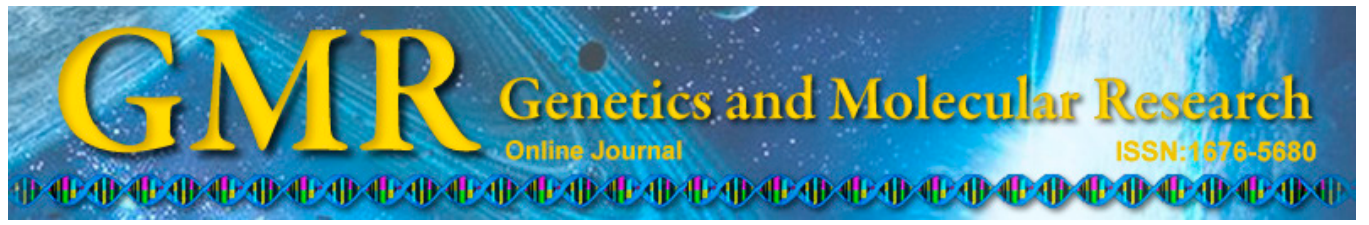

\title{
Characterization of a TIR-NBS-LRR gene associated with downy mildew resistance in grape
}

\author{
J.J. Fan ${ }^{1 *}$, P. Wang ${ }^{2 *}$, X. Xu' ${ }^{1}$, K. Liu ${ }^{3}$, Y.Y. Ruan ${ }^{1}$, Y.S. Zhu ${ }^{1}$, Z.H. Cui ${ }^{1}$ \\ and L.J. Zhang ${ }^{1}$ \\ ${ }^{1}$ College of Bioscience and Technology, Shenyang Agricultural University, \\ Shenyang, China \\ ${ }^{2}$ Innovation Center, Liaoning Academy of Agricultural Sciences, \\ Shenyang, China \\ ${ }^{3}$ Heilongjiang Academy of Agricultural Sciences, Haerbin, China \\ *These authors contributed equally to this study. \\ Corresponding author: J.J. Fan \\ E-mail: jinjuanf@hotmail.com
}

Genet. Mol. Res. 14 (3): 7964-7975 (2015)

Received September 29, 2014

Accepted April 28, 2015

Published July 17, 2015

DOI http://dx.doi.org/10.4238/2015.July.17.4

\begin{abstract}
Grapevine downy mildew, caused by Plasmopara viticola, is a devastating disease that results in considerable economic losses as well as environmental damage through the repeated application of fungicides. The nucleotide-binding site leucine-rich repeat gene family functions in plant immunoactivity against various pathogens and pests. In this study, the $5^{\prime}$ and $3^{\prime}$ ends of the resistance gene homology fragment $R G A 5$ were obtained by rapid amplification of cDNA ends. The 4282-base pair full-length cDNA was obtained using gene-specific primers, and the corresponding 1335-amino acid protein sequence contained characteristic nucleotide-binding site leucine-rich repeat domains of plant resistance proteins, including the toll-interleukin receptor type region. Expression of $R G A 5$ during $P$. viticola infection and abiotic stress was investigated using quantitative
\end{abstract}


real-time polymerase chain reaction. The results showed that treatment with $P$. viticola and 4 abiotic stimuli (salicyclic acid, methyl-jasmonate, abscisic acid, $\mathrm{H}_{2} \mathrm{O}_{2}$ ) significantly induced $R G A 5$ within 12 days of inoculation. Therefore, $R G A 5$ may play a critical role in protecting grapevines against $P$. viticola via signaling pathways involving these molecules.

Key words: Downy mildew; Nucleotide-binding site leucine-rich repeat; Plasmopara viticola; Rapid amplification of cDNA ends; Quantitative real-time polymerase chain reaction; Vitis spp

\section{INTRODUCTION}

Downy mildew, caused by the oomycete Plasmopara viticola (Berk. and Curt.) Berl. and de Toni, is the most economically important fungal disease of grapes (Vitis spp). This devastating disease causes partial or total crop losses and has a severe environmental impact resulting from repeated fungicide applications required as a control measure. Genetic modification of grapevines to confer resistance to downy mildew would be of considerable economic and environmental benefit.

Recently, genes that confer resistance $(R)$ to different types of pathogens, including viruses, bacteria, fungi, and nematodes, have been cloned using map-based cloning and transposon tagging procedures (Dangl and Jones, 2001; Martin et al., 2003; Meyers et al., 2005). With the exception of Hml and Mlo (Johal and Briggs, 1992; Buschges et al., 1997), R genes are highly structurally conserved and include leucine zipper, nucleotide binding site (NBS), leucine-rich repeat (LRR), transmembrane, and serine/threonine protein kinase domains (Dan$\mathrm{gl}$ and Jones, 2001). The NBS-LRR gene family is the largest, and members are characterized by an N-terminal coiled-coil or toll-interleukin receptor type (TIR) domain, an NBS domain and an extended domain of LRR (Bent and Mackey, 2007).

The products of $R$ genes act as immune receptors that directly or indirectly sense the presence of a pathogen and trigger strong defense responses, which frequently result in cell death (Jones and Dangl, 2006) and completely halt further pathogen growth. $R$ gene-mediated resistance operates through a few conserved signaling pathways and orchestrates the coordinated activation of defense genes. Within each species, diverse $R$ genes may require distinct signaling pathways and recruit particular subclasses of pathogenesis-related $(P R)$ genes (Hammond-Kosack and Jones, 1996).

Three main phytohormones have been established as essential components mediating the host responses triggered by pathogens, including salicylic acid (SA), jasmonic acid (JA), and ethylene. The SA-dependent signaling pathways typically converge to execute the cell death-like hypersensitive response and synthesis of PR-1 proteins, which effectively halt biotrophs such as $P$. viticola (Feys et al., 2001). JA/ethylene defense pathways are more commonly associated with resistance to necrotrophs and response to wounding, general elicitors, and non-host pathogens (Zimmerli et al., 2004). However these generalities are disputed in grape, as methyl-jasmonate (MeJA) levels increase in response to P. viticola, and the application of exogenous MeJA to the leaf lamina alone triggers cysteine protease-associated cell death (Polesani et al., 2010). The persistence of defense responses and disease outcome are determined by complex networks of interactions between multiple hormone signaling path- 
ways (Santner and Estelle, 2009). SA- and JA/ethylene-mediated signaling pathways are more tightly connected than initially predicted, and may not be antagonistic (Mur et al., 2006).

Recent studies have indicated that other hormones such as abscisic acid (ABA) are also involved in plant defense signaling pathways. In addition, $\mathrm{H}_{2} \mathrm{O}_{2}$ has been confirmed as a second messenger that activates defense gene expression (Orozco-Cárdenas et al., 2001) and is one of the earliest plant responses triggered during incompatible interactions between pathogens and plants (Zhu-Salzman et al., 2004).

Complete determination of the Vitis vinifera genome sequence (both highly homozygous and heterozygous varieties) has led to the identification of putative resistance genes and defense signaling elements (Casagrande et al., 2011). With the introduction of deep sequencing, a complex gene family encoding NBS-LRR proteins was identified, and its members have been isolated and characterized (Seehalak et al., 2011). However, full functional characterization of Vitis resistance gene analogs (RGAs) has not been widely reported.

To identify grapevine RGAs that are potentially involved in host defense responses against $P$. viticola infection, we characterized NBS-LRR family RGAs and investigated their transcription (Wang et al., 2013). Vitis RGA5 was further analyzed and the flanking sequence of $R G A 5$ was obtained using specific primers with rapid amplification of cDNA ends (RACE). $R G A 5$ expression during $P$. viticola infection and treatment with $\mathrm{SA}, \mathrm{MeJA}, \mathrm{ABA}$, and $\mathrm{H}_{2} \mathrm{O}_{2}$ was further investigated using quantitative real-time-polymerase chain reaction (qRT-PCR).

\section{MATERIAL AND METHODS}

\section{Plant material, pathogen infection, and hormone treatments}

Two different Vitis species, the resistant Vitis riparia cv Beta and the susceptible Vitis vinifera cv Thompsons Seedless, were used in this study. One-year-old grapevines obtained from a commercial nursery were grown in a greenhouse at $20^{\circ}-30^{\circ} \mathrm{C}$ and $70-85 \%$ relative humidity.

The $P$. viticola isolate used in this study, which was collected from 'kyoho' at the Experimental Research Station at the Liaoning Academy of Agricultural Sciences, was obtained from a single infected leaf. The detached leaf exhibited oil spots and was thoroughly washed under running tap water to remove the sporangia, and then incubated in a humid chamber at room temperature overnight to induce sporulation. On the following day, fresh sporangia were collected with a brush and used to inoculate the same cultivar, which was then and maintained under greenhouse conditions until sporulation.

For pathogen infection and hormone treatment, 1-year-old grapevines were treated with $P$. viticola and signaling molecules, including $2 \mathrm{mM}, 100 \mu \mathrm{M}$ MeJA, $100 \mu \mathrm{M}$ ABA. For $\mathrm{H}_{2} \mathrm{O}_{2}$ treatment, the same seedlings were sprayed with $10 \mu \mathrm{M} \mathrm{H}_{2} \mathrm{O}_{2}$ in sterile water. Control plants were sprayed with sterile water only. Plasmopara viticola infections were initiated by spraying the $3 \mathrm{rd}$ and 4 th grapevine leaves with a suspension containing approximately 10,000 sporangia/mL. Leaves were covered with plastic bags overnight to increase the humidity, and plants were maintained in the greenhouse at $23^{\circ} \mathrm{C}$ under a 16 -h: 8 -h (light:dark) photoperiod.

\section{RNA extraction and cDNA synthesis}

Total RNA was extracted from grapevine leaves using an improved Trizol method (Que et al., 2008) and treated with DNase I to remove genomic DNA contamination. Next, 
DNase-treated RNA was reverse transcribed (RT) using the SuperScript First-Strand Synthesis System for an RT-PCR Kit (Invitrogen, Carlsbad, CA, USA). An RT-PCR reaction lacking the reverse transcriptase enzyme was performed during the cDNA synthesis reaction to test whether each mRNA sample contained genomic DNA contamination.

\section{RACE}

The $3^{\prime}$ and $5^{\prime}$ ends of $R G A 5$ were determined using RACE-PCR using the SMART RACE cDNA amplification kit (Clontech, Mountain View, CA, USA), according to manufacturer instructions. Oligonucleotide primers based on the RGA5 sequence (Wang et al., 2013) and the adaptor included in the kit were used for amplification and sequencing. The forward primers $R G A 5 \mathrm{~F} 1$ and $R G A 5 \mathrm{~F} 2$ were used as gene-specific primers in 3'-RACE-PCR. Primer $R G A 5 \mathrm{~F} 1+$ adaptor were used generate the first amplicons, followed by semi-nested PCR with primer $R G A 5 \mathrm{~F} 2$ + adaptor. A similar strategy with reverse primers $R G A 5 \mathrm{R} 1$ and $R G A 5 \mathrm{R} 2$ was used for 5'-RACE- PCR, with primer $R G A 5 \mathrm{R} 1+$ adaptor to produce the first amplicons, followed by semi-nested PCR with primer pair $R G A 5 \mathrm{R} 2$ + adaptor. Specific full-length primers were designed based on the split full-length sequence according to the open reading frame (ORF) (Table 1). All oligonucleotide primers were designed using the Primer 3.0 software.

Table 1. Sequence of primers used for RACE and full-length sequence.

\begin{tabular}{llc}
\hline Primer name & $5^{\prime} \rightarrow 3^{\prime}$ sequence & Sequence length (bp) \\
\hline$R G A 5 \mathrm{~F} 1$ & ATAGGCATGGTACAGAA & 17 \\
$R G A 5 \mathrm{~F} 2$ & TTAGGCAGCTATGTGGC & 17 \\
$R G A 5 \mathrm{R} 1$ & GTGTAGATTCGCTCATA & 17 \\
$R G A 5 \mathrm{R} 2$ & CTTTAGCAATGGTAGTC & 17 \\
$R G A 5 \mathrm{FullF}$ & GTCTGGACGAGCTAGTAAA & 19 \\
RGA5FullR & CACTCTGATGCCTGAAC & 17 \\
\hline
\end{tabular}

\section{Cloning and sequence analysis}

PCR products were resolved by $1.5 \%$ agarose gel electrophoresis and stained with ethidium bromide. Target bands were excised and purified using a QIAquick Gel Extraction Kit (Qiagen, Hilden, Germany), cloned into the pGEM-T Easy vector (Promega, Madison, WI, USA), and transformed into Escherichia coli DH5 $\alpha$. Positive clones identified by PCR and enzyme digestion were sequenced (Sangon Biotech, Shanghai, China).

The full-length cDNA was assembled and examined in BLASTN to search the NCBI database in order to identify all sequences in the contig showing similarity to characterized sequences. The genomic DNA sequences of $R G A 5$ clones were scanned for putative protein coding regions using the Genoscope database, which contains grapevine genome sequencing results. ORF predictions were performed using FGENESH 2.6 (Salamov and Solovyev, 2000), and GENSCAN (Burge and Karlin, 1997). Candidate ORFs were searched against BLASTX in the NCBI database, and analyzed using the NCBI conserved domain tools. Pairwise comparisons and multiple alignments of nucleotide and deduced amino acid sequences were performed using the ClustalX software (Thompson et al., 1997). 


\section{Design of primers for expression analysis}

Based on the RGA nucleotide sequences, gene-specific primer pairs were designed using the Primer 3.0 software. Glyceraldehyde-3-phosphate dehydrogenase (XM_ 002263109) was used as a reference gene for data normalization (Selim et al., 2012) (Table $\overline{2}$ ).

Table 2. Sequence of primers used for qRT-PCR in grapevine.
\begin{tabular}{lllll}
\hline Gene & Forward primer $\left(5^{\prime}\right.$ to $\left.3^{\prime}\right)$ & Reverse primer $\left(5^{\prime}\right.$ to $\left.3^{\prime}\right)$ & Tm $\left({ }^{\circ} \mathrm{C}\right)$ & Accession Nos. \\
\hline$R G A 5$ & GAAGTCTCACATTAGAAT & TGGTATTGATGACATAAG & 59 & AGF69193 \\
GAPDH & ATCAAGGAGGAGTCAGAG & GTTGTCACCGATGAAGTC & 60 & XM_002263109 \\
\hline
\end{tabular}

\section{Quantitative real-time PCR and data analysis}

For qRT-PCR, reactions were carried out on 96-well plates using the Bio-Rad CFX96 Real-Time PCR System and the Bio-Rad CFX96 Manager Software (Bio-Rad, Hercules, CA, USA) using the SYBR Green-based PCR assay. Each $20 \mu \mathrm{L}$ reaction contained $5 \mu \mathrm{L}$ diluted cDNAs, $10 \mu \mathrm{L} 2 \mathrm{X}$ SYBRGreen PCR MasterMix (TaKaRa, Shiga, Japan), and $0.25 \mu \mathrm{M}$ of each primer, and thermal cycling was performed as follows: $95^{\circ} \mathrm{C}$ for $3 \mathrm{~min}$, followed by 40 cycles at $95^{\circ} \mathrm{C}$ for $15 \mathrm{~s}, 60^{\circ} \mathrm{C}$ for $30 \mathrm{~s}$, and $72^{\circ} \mathrm{C}$ for $15 \mathrm{~s}$. Melting curves were analyzed between $55^{\circ}$ $95^{\circ} \mathrm{C}$ after 40 cycles. The grape glyceraldehyde-3-phosphate dehydrogenase gene was used to normalize the data. In addition, a reverse transcription negative control was included to check for potential genomic DNA contamination. Each qRT-PCR analysis was performed in triplicate and the mean was used for qRT-PCR analysis. The relative expression of the $R$ gene was calculated using the $2^{-\Delta \Delta C t}$ method (Livak and Schmittgen, 2001). The threshold cycle (CT) values for both the target and internal control genes were calculated using the means of 3 independent PCRs. Data were analyzed using the Bio-Rad CFX96 Manager Software and Microsoft Excel 2007.

\section{RESULTS}

\section{RACE-PCR amplification of an $R G A 5$ gene fragment}

Degenerate oligonucleotides were used to clone the full-length cDNA of grape RGA5. PCR with the primer pair $R G A 5 \mathrm{~F} 1+R G A 5 \mathrm{R} 1$ and genomic DNA amplified a product approximately 328 base pair (bp) in length. The product was sequenced using the primer pair $R G A 5 \mathrm{~F} 2+R G A 5 \mathrm{R} 2$. The 3'- and 5'-termini were elucidated by RACE-PCR using RNA from infected leaves as a template for full-length cDNA synthesis. Sequence comparison of the 2 RACE-PCR products with the internal amplicon showed 100\% identity in the overlapping regions, and the 3 ' product contained a poly-A motif at the 3'-terminus, which resulted in the identification of a 4008-bp ORF (Figure 1).

\section{Structure and characterization of full-length $R G A 5$}

Sequence comparison of the full-length cDNA and the results from the grapevine genome sequencing project indicated that the transcribed portion of the $R G A 5$ was $5382 \mathrm{bp}$, 
including a region of $1.1 \mathrm{~kb}$ that contained a promoter sequence upstream of the ATG start codon, a 130 bp 3'-untranslated region, and a 4008-bp coding sequence. The 4008-bp coding sequence encoded a protein of 1335 amino acids and included the characteristic domains of an NBS-LRR family protein (Figure 2). The conserved NBS motifs (P-loop, kinase-2, kinase-3, glucagon-like peptide-1, and large hydrophilic domain) were highly similar to those of other NBS-LRR resistance proteins (Traut, 1994; Grant et al., 1995). Significant homology to the $\mathrm{N}$-terminus of the Drosophila Toll and mammalian TIR indicate that $R G A 5$ belonged to the TIR subfamily of NBS-LRR resistance protein. The LRR domain consisted of 21 repeats of the consensus motif LxxLxLxx (Kobe and Deisenhofer, 1994).

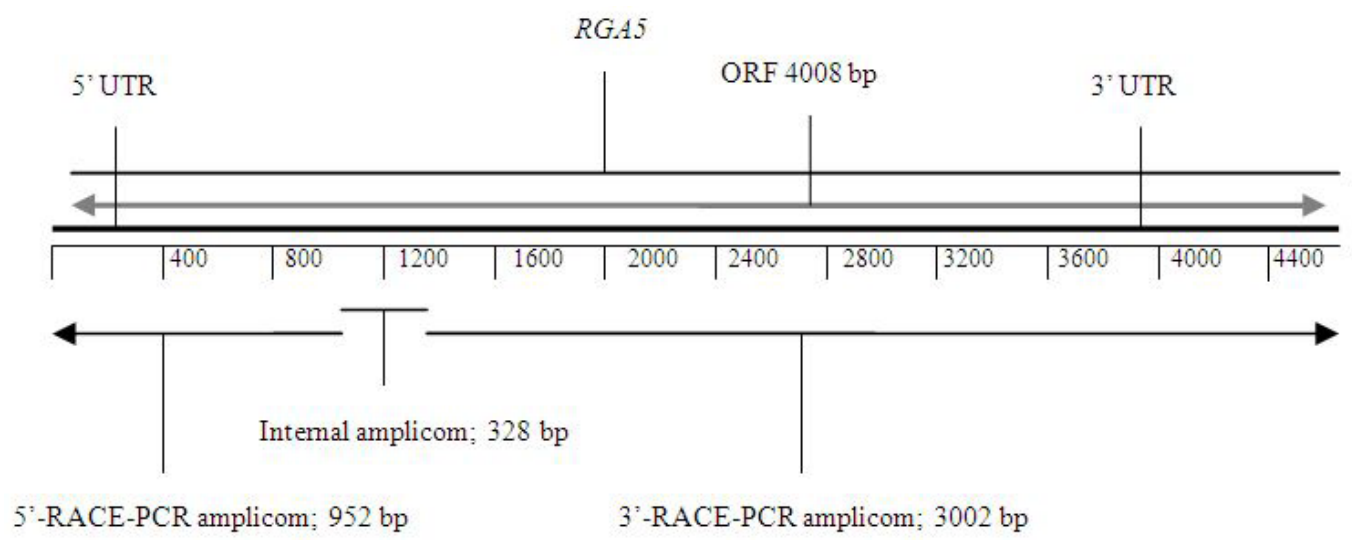

Figure 1. Scheme of PCR experiments. The alignment shows the 3 amplicons, which led to identification of the $\mathrm{ORF}$ and untranslated regions of RGA5. The internal fragment was amplified using primer pairs F1 + R1 and F2 $+\mathrm{R} 2$ with nested PCR. The 3'-RACE-PCR fragment was amplified using the gene-specific primers $R G A 5 \mathrm{~F} 1$ and $R G A 5 \mathrm{~F} 2$ with semi-nested PCR. The 5'-RACE-PCR product was amplified using gene-specific primers $R G A 5 \mathrm{R} 1$ and RGA5R2 with semi-nested PCR. In all RACE experiments, the adaptor primer supplied in the SMART RACE cDNA amplification kit was used.

\section{Expression of $R G A 5$ during compatible and incompatible interactions with P. viticola}

To characterize $R G A 5$ transcription, the induction of $R G A 5$ expression in response to $P$. viticola was examined by qRT-PCR. Grape and related species exhibit a wide spectrum of resistance to this biotrophic pathogen. Two different Vitis species, the resistant V. riparia cv Beta and the susceptible $V$. vinifera cv Thompsons Seedless, were challenged with $P$. viticola (or water as a control). RGA5 expression following inoculation was compared with $\mathrm{H}_{2} \mathrm{O}$ inoculated leaves.

Twelve days after inoculation with $P$. viticola, a number of oil spots were observed on leaves of the resistant $V$. riparia, whereas sporangia covered nearly the entire leaf surface of the susceptible $V$. vinifera (data not shown). RGA5 transcription was found to be 1.5 -fold higher in $V$. riparia 2 days after inoculation, with transcription peaking (5.9-fold) at 6 days. $R G A 5$ expression then decreased to 4.6 -fold at 8 days and 3.1-fold at 12 days. In contrast, $R G A 5$ transcription in $V$. vinifera peaked at 2 days (3.5-fold) and decreased to 2.2 -fold at 8 days (Figure $3 \mathrm{~A}$ ). These results suggest that $R G A 5$ is involved in the host defense response against $P$. viticola. 


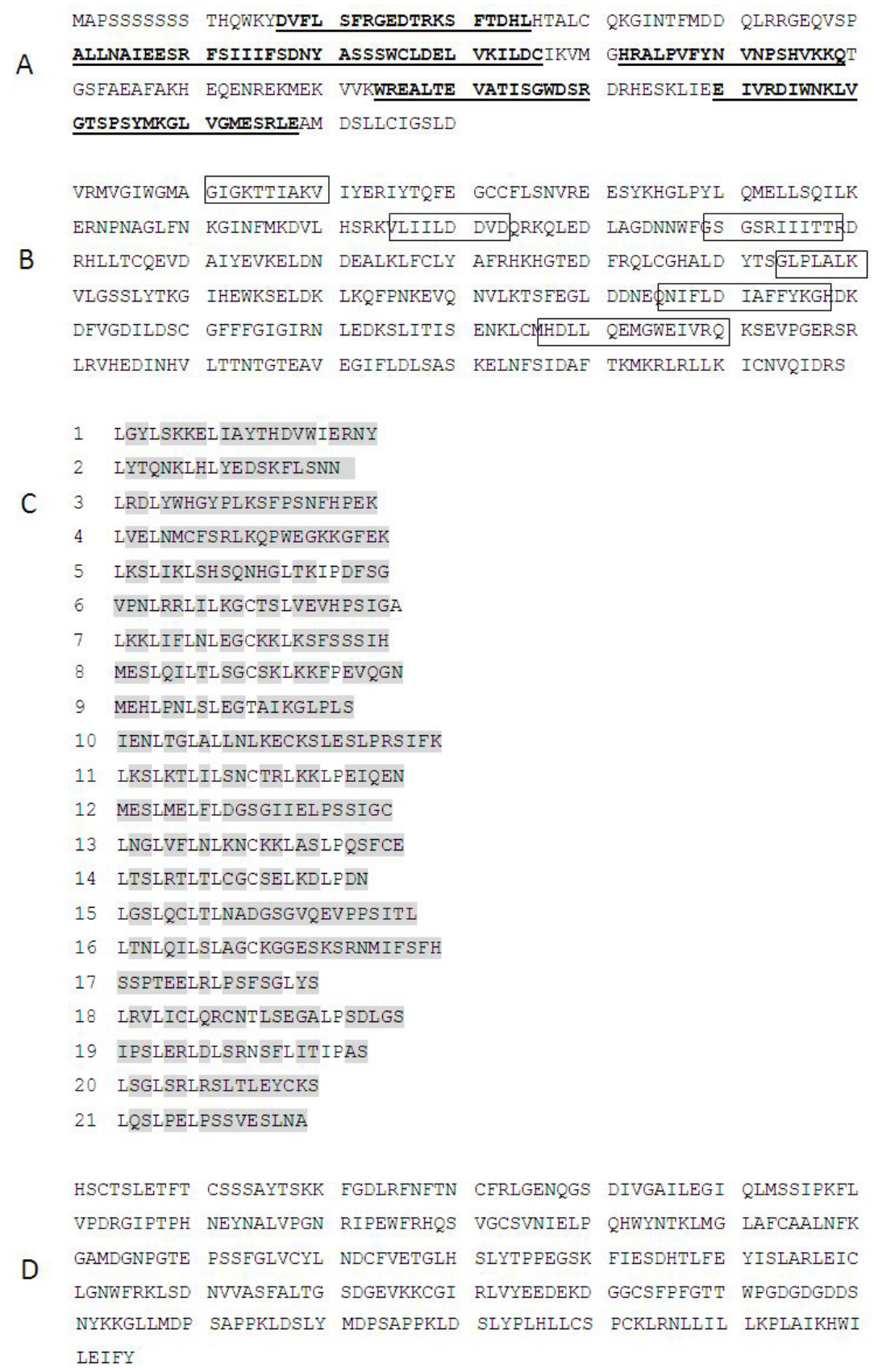

Figure 2. Amino acid sequence of full-length RGA5, showing the 5 domains: A. N-terminus Drosophila TIR (Meyers et al., 2003); B. NBS, P-loop, GGIGKTT; kinase-2, VLDD; kinase-3a, GSR/KILVTTR; HD, GLPLAL (Meyers et al., 2003) (in boxed); C. leucine-rich repeat (LRR; shaded areas); and D. C-terminal region. Alignment of the LRR domain highlights the LxxLxxLxLxx (N/C/T)x(x)LxxIPxxAxx repeat (Jones and Jones, 1997), with conserved amino acids in bold. 

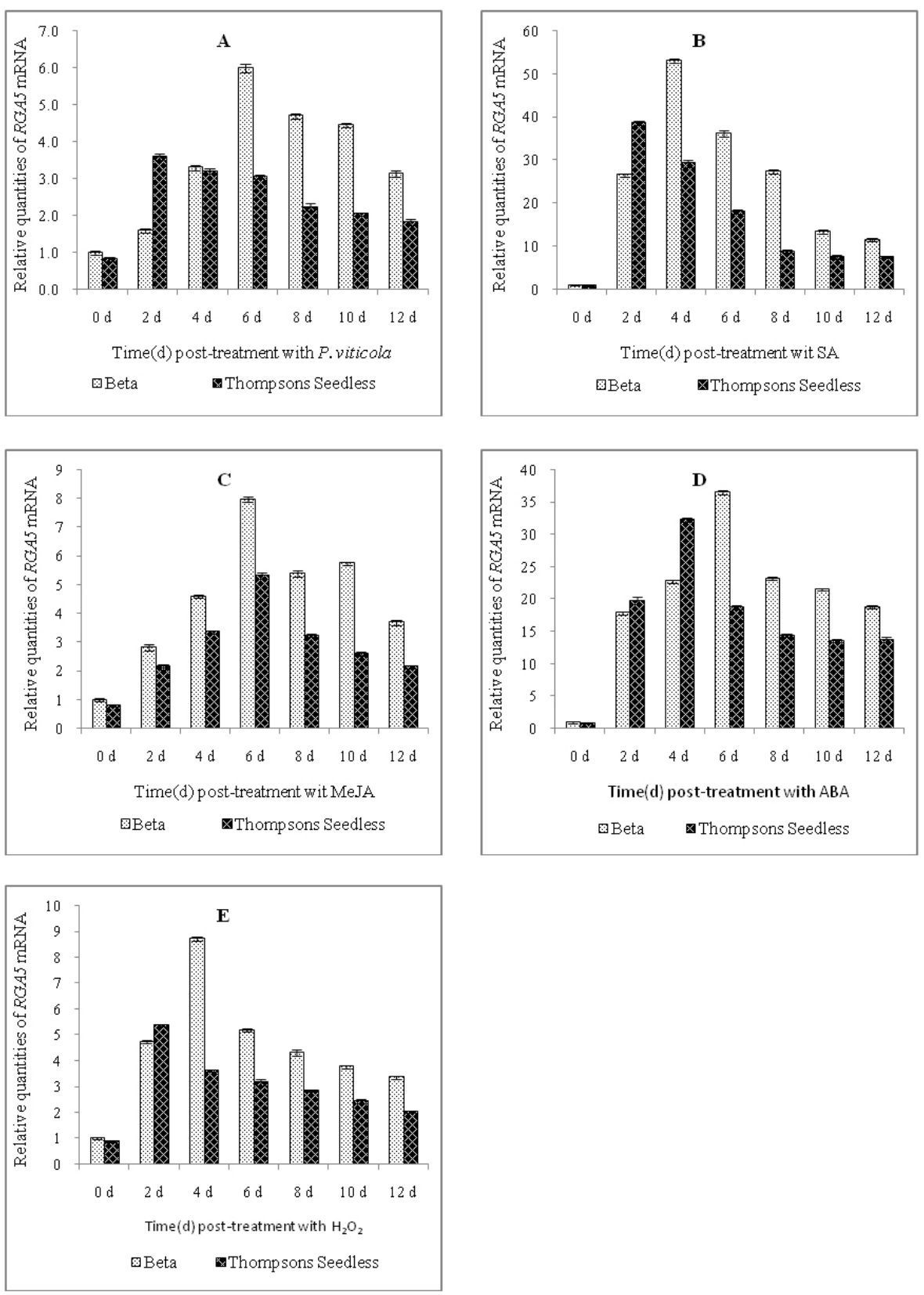

Figure 3. Upregulation of RGA5 expression in response to Plasmopara viticola and defense signaling molecules. (A) RGA5 transcription in response to P. viticola infection for $0,2,4,6,8,10$, and 12 days in Vitis riparia and V. vinifera. (B) RGA5 expression response to exogenously applied hormones and $\mathrm{H}_{2} \mathrm{O}_{2}$ measured by qRT-PCR. Seedlings were treated using (B) SA, (C) MeJA, (D) ABA, and (E) $\mathrm{H}_{2} \mathrm{O}_{2}$. Expression levels are relative to levels in $V$. riparia seedlings that were not inoculated at day 0 after normalization against grape glyceraldehyde 3-phosphate dehydrogenase. Data shown are means and standard errors of 3 independent qRT-PCR experiments. 


\section{Induction of $R G A 5$ expression in response to defense signaling molecules}

To evaluate whether the expression of $R G A 5$ was induced by a combination of $P$. viticola infection and known resistance-inducing chemicals, $R G A 5$ transcription following exogenous application of $\mathrm{SA}, \mathrm{MeJA}, \mathrm{ABA}$, and $\mathrm{H}_{2} \mathrm{O}_{2}$ was examined by qRT-PCR analysis.

Following SA treatment, $R G A 5$ transcription was upregulated by 26 -fold at 2 days after treatment in $V$. riparia, with expression peaking at 53-fold after 4 days. Transcription then decreased sharply, but was still upregulated (11-fold) at 12 days post-treatment (Figure 3B). For $V$. vinifera, $R G A 5$ transcription peaked at 2 days after treatment (38-fold), and then decreased to 7.5 -fold by day 12 .

Exposure to MeJA also upregulated $R G A 5$ transcription in $V$. riparia, by 2.8 -fold at day 2 , with expression peaking at 8 -fold by day 6 . However, transcription then decreased to 3.7 -fold by day 12 (Figure 3C). Similarly, upregulation of $R G A 5$ transcription in $V$. vinifera was short-lived, and peaked by 5.3 -fold at 6 days after treatment.

$R G A 5$ transcription in $V$. riparia was induced rapidly after treatment with ABA (Figure 3D), reaching 17-fold at 2 days, peaking at 6 days (36-fold), and then decreasing to 18 -fold at 12 days after treatment. In $V$. vinifera, transcription peaked at 4 days (32-fold) and decreased to 13 -fold by day 12 .

In response to $\mathrm{H}_{2} \mathrm{O}_{2}$ treatment, $R G A 5$ expression in $V$. riparia was upregulated to a lesser extent, peaking at 8.7 -fold higher than baseline levels at 4 days after treatment, before steadily decreasing (Figure 3E). In V. vinifera, expression was increased by 5.3 -fold on day 2 , then decreased to 2.4 -fold by day 12 .

These results clearly demonstrated strong induction of RGA5 expression by $P$. viticola infection, and this response was enhanced by exogenous application of SA, MeJA, ABA, and $\mathrm{H}_{2} \mathrm{O}_{2}$, suggesting that $R G A 5$ may function in host defense responses involving signaling pathways that include these molecules.

\section{DISCUSSION}

Most resistance genes that have been cloned over the last 2 decades belong to the NBS-LRR family, and proteins with NBS and LRR domains are associated exclusively with plant disease resistance (Lukasik and Takken, 2009). The NBS-LRR domain architecture indicates a role in pathogen recognition and defense response signaling (Ameline-Torregosa et al., 2008). NBS-encoding genes are widely distributed in diverse plant genomes, and 341 NBS genes (including 84 coiled-coil-NBS-LRR and 37 TIR-NBS-LRR genes) have been identified in the grape genome (Velasco et al., 2007).

Using homology cloning approaches, we previously identified a number of homologous disease-resistant gene fragments (Wang et al., 2013). In order to obtain the full-length gene sequences, 2 methods are typically used; gene library screening and RACE. The latter is a simpler and more effective technique for amplifying $5^{\prime}$ and 3 ' ends of low-abundance gene fragments and for cloning full-length genes or identifying novel genes.

In this study, we determined the full-length sequence of $R G A 5$, and NB-ARC domains including the P-loop, kinase 2, kinase 3, glucagon-like peptide-1, and LHD conserved motifs were identified using the NCBI conserved domain tools. The 2 major NBS-LRR subfamilies can be distinguished by either the presence or absence of an amino-terminal TIR-like domain (Meyers et al., 1999). Non-TIR subgroup NBS-LRR genes are common, and most include a 
coiled-coil or leucine zipper domain (Bai et al., 2002). The $R G A 5$ gene contains a TIR domain, as do many other NBS-LRRs. The N-terminal LRR domain of RGA5 includes 21 consensus LxxLxLxx repeats (Figure 2). Therefore, RGA5 belongs to the TIR-NBS-LRR class of resistance-related genes.

Most plant resistance genes are transcriptionally regulated in response to pathogen attack. In the present study, RGA5 transcription was investigated to gain insight into its possible involvement in host defense responses (Figure 3A). In the resistant $V$. riparia cv Beta and the susceptible $V$. vinifera cv Thompsons Seedless, infection with $P$. viticola significantly enhanced expression of $R G A 5$, suggesting a correlation between $R G A 5$ and resistance to $P$. viticola and indicating a role in $P$. viticola-induced defense responses. Similar expression patterns have been observed for Pib, a blast resistance gene in rice (Wang et al., 1999, 2001), and $H s 1^{\text {pro-l }}$, a downy mildew resistance gene in Cucumis (Wan et al., 2010).

Previous studies reported that signaling molecules not only communicate downstream resistance events, but also upregulate the expression of $R$ genes. In Arabidopsis, SA treatment induces the expression of SSI4, a putative TIR-NBS-LRR class $R$ gene, as well as the closely related TIR-NBS-LRR genes RPP1 and RPS4 (Shirano et al., 2002). In grape, of all VvMLO genes tested, $V v M L O 8$ and $V v M L O 10$ were upregulated by over 50 -fold following $\mathrm{H}_{2} \mathrm{O}_{2}$ treatment, while $V v M L O 4, V v M L O 6$ and $V v M L O 10$ showed the most marked response to SA treatment (Feechan et al., 2008).

In this study, $R G A 5$ was upregulated by $P$. viticola, as well as by the defense-signaling molecules MeJA, ABA, and $\mathrm{H}_{2} \mathrm{O}_{2}$, in addition to SA (Figure 3B-E). This suggests that $R G A 5$ plays a role in mediating the cross-talk between defense-signaling pathways. Because both SA and MeJA stimulated RGA5 expression, these molecules may have a synergistic role in mediating defense responses in grape. Interestingly, resistant $V$. riparia $\mathrm{cv}$ Beta and susceptible $V$. vinifera cv Thompsons Seedless displayed distinct $R G A 5$ transcription patterns. Upregulation of $R G A 5$ transcription by MeJA peaked at an 8 -fold increase by day 6 in $V$. riparia, whereas stimulation by SA peaked at 53 -fold by day 4 . This suggests that $R G A 5$ is involved in different defense responses during different stages. Importantly, RGA5 expression was upregulated to much higher levels in $V$. riparia, which may be related to downy mildew sensitivity in $V$. vinifera and other sensitive cultivars.

In conclusion, $R G A 5$ is activated in both resistant $V$. riparia and susceptible $V$. vinifera, cultivars during infection by $P$. viticola and functions in signal transduction pathways that are mediated by $\mathrm{SA}, \mathrm{MeJA}, \mathrm{ABA}$, and $\mathrm{H}_{2} \mathrm{O}_{2}$. The potentially prominent role of $R G A 5$ in grape defense responses warrants further investigation.

\title{
ACKNOWLEDGMENTS
}

\author{
Research supported by the Agro-Scientific Research in the Public Interest \\ (\#201203035).
}

\section{REFERENCES}

Ameline-Torregrosa C, Wang BB, O'Bleness MS, Deshpande S, et al. (2008). Identification and characterization of nucleotide-binding site-leucine-rich repeat genes in the model plant Medicago truncatula. Plant Physiol. 146: 5-21.

Bai J, Pennill LA, Ning J, Lee SW, et al. (2002). Diversity in nucleotide binding site-leucine-rich repeat genes in cereals. Genome Res. 12: 1871-1884.

Bent AF and Mackey D (2007). Elicitors, effectors, and R genes: the new paradigm and a lifetime supply of questions. 
Annu. Rev. Phytopathol. 45: 399-436.

Burge C and Karlin S (1997). Prediction of complete gene structures in human genomic DNA. J. Mol. Biol. 268: 78-94.

Buschges R, Hollricher K, Panstruga R, Simons G, et al. (1997). The barley Mlo gene: a novel control element of plant pathogen resistance. Cell 88: 695-705.

Casagrande K, Falginella L, Castellarin SD, Testolin R, et al. (2011). Defence responses in Rpv3-dependent resistance to grapevine downy mildew. Planta 234: 1097-1109.

Dangl JL and Jones JD (2001). Plant pathogens and integrated defence responses to infection. Nature 411: 826-833.

Feechan A, Jermakow AM, Torregrosa L, Panstruga R, et al. (2008). Identification of grapevine $M L O$ gene candidates involved in susceptibility to powdery mildew. Funct. Plant Biol. 35: 1255-1266.

Feys BJ, Moisan LJ, Newman MA and Parker JE (2001). Direct interaction between the Arabidopsis disease resistance signaling proteins, EDS1 and PAD4. EMBO J. 20: 5400-5411.

Grant MR, Godiard L, Straube E, Ashfield T, et al. (1995). Structure of the Arabidopsis RPM1 gene enabling dual specificity disease resistance. Science 269: 843-846.

Hammond-Kosack KE and Jones JD (1996). Resistance gene-dependent plant defense responses. Plant Cell 8: 1773-1791.

Johal GS and Briggs SP (1992). Reductase activity encoded by the HM1 disease resistance gene in maize. Science 258: 985-987.

Jones DA and Jones JDG (1997). The role of leucine-rich repeat proteins in plant defences. Adv. Bot. Res. 24: 89-167.

Jones JD and Dangl JL (2006). The plant immune system. Nature 444: 323-329.

Kobe B and Deisenhofer J (1994). The leucine-rich repeat: a versatile binding motif. Trends Biochem. Sci. 19: 415-421.

Livak KJ and Schmittgen TD (2001). Analysis of relative gene expression data using real-time quantitative PCR and the 2(-Delta Delta C(T)) method. Methods 25: 402-408.

Lukasik E and Takken FL (2009). STANDing strong, resistance proteins instigators of plant defence. Curr. Opin. Plant Biol. 12: 427-436.

Martin GB, Bogdanove AJ and Sessa G (2003). Understanding the functions of plant disease resistance proteins. Annu. Rev. Plant Biol. 54: 23-61.

Meyers BC, Dickerman AW, Michelmore RW, Sivaramakrishnan S, et al. (1999). Plant disease resistance genes encode members of an ancient and diverse protein family within the nucleotide-binding superfamily. Plant J. 20: 317-332.

Meyers BC, Kozik A, Griego A, Kuang H, et al. (2003). Genome-wide analysis of NBS-LRR-encoding genes in Arabidopsis. Plant Cell 15: 809-834.

Meyers BC, Kaushik S, Nandety RS (2005). Evolving disease resistance genes. Curr. Opin. Plant Biol. 8: 129-134.

Mur LA, Kenton P, Atzorn R, Miersch O, et al. (2006). The outcomes of concentration-specific interactions between salicylate and jasmonate signaling include synergy, antagonism, and oxidative stress leading to cell death. Plant Physiol. 140: 249-262.

Orozco-Cárdenas ML, Narváez-Vásquez J and Ryan CA (2001). Hydrogen peroxide acts as a second messenger for the induction of defense genes in tomato plants in response to wounding, systemin, and methyl jasmonate. Plant Cell 13: 179-191.

Polesani M, Bortesi L, Ferrarini A, Zamboni A, et al. (2010). General and species-specific transcriptional responses to downy mildew infection in a susceptible (Vitis vinifera) and a resistant (V. riparia) grapevine species. BMC Genomics 11: 117.

Que YX, Li W, Xu JS, Xu LP, et al. (2008). A simple and versatile protocol for isolation of RNA from plant, fungi and animal. J. Agric. Sci. Tech-Iran 2: 63-65.

Salamov AA and Solovyev VV (2000). Ab initio gene finding in Drosophila genomic DNA. Genome Res. 10: 516-522.

Santner A and Estelle M (2009). Recent advances and emerging trends in plant hormone signalling. Nature 459: 10711078.

Seehalak W, Moonsom S, Metheenukul P and Tantasawat P (2011). Isolation of resistance gene analogs from grapevine resistant and susceptible to downy mildew and anthracnose. Sci. Hortic.-Amsterdam 128: 357-363.

Selim M, Legay S, Berkelmann-Lohnertz B, Langen G, et al. (2012). Identification of suitable reference genes for realtime RT-PCR normalization in the grapevine-downy mildew pathosystem. Plant Cell Rep. 31: 205-216.

Shirano Y, Kachroo P, Shah J and Klessig DF (2002). A gain-of-function mutation in an Arabidopsis Toll Interleukin1 receptor-nucleotide binding site-leucine-rich repeat type $\mathrm{R}$ gene triggers defense responses and results in enhanced disease resistance. Plant Cell 14: 3149-3162.

Thompson JD, Gibson TJ, Plewniak F, Jeanmougin F, et al. (1997). The CLUSTAL_X windows interface: flexible strategies for multiple sequence alignment aided by quality analysis tools. Nucleic Acids Res. 25: 4876-4882.

Traut TW (1994). The functions and consensus motifs of nine types of peptide segments that form different types of nucleotide-binding sites. Eur. J. Biochem. 222: 9-19.

Velasco R, Zharkikh A, Troggio M, Cartwright DA, et al. (2007). A high quality draft consensus sequence of the genome 
of a heterozygous grapevine variety. PLoS One 2: e1326.

Wan H, Zhao Z, Malik AA, Qian C, et al. (2010). Identification and characterization of potential NBS-encoding resistance genes and induction kinetics of a putative candidate gene associated with downy mildew resistance in Cucumis. BMC Plant Biol. 10: 186.

Wang P, Liu CY, Wang DX, Liang CH, et al. (2013). Isolation of resistance gene analogs from grapevine resistant to downy mildew. Sci. Hortic.-Amsterdam 150: 326-333.

Wang ZX, Yano M, Yamanouchi U, Iwamoto M, et al. (1999). The Pib gene for rice blast resistance belongs to the nucleotide binding and leucine-rich repeat class of plant disease resistance genes. Plant J. 19: 55-64.

Wang ZX, Yamanouchi U, Katayose Y, Sasaki T, et al. (2001). Expression of the Pib rice-blast-resistance gene family is up-regulated by environmental conditions favouring infection and by chemical signals that trigger secondary plant defences. Plant Mol. Biol. 47: 653-661.

Zhu-Salzman K, Salzman RA, Ahn JE and Koiwa H (2004). Transcriptional regulation of sorghum defense determinants against a phloem-feeding aphid. Plant Physiol. 134: 420-431.

Zimmerli L, Stein M, Lipka V, Schulze-Lefert P, et al. (2004). Host and non-host pathogens elicit different jasmonate/ ethylene responses in Arabidopsis. Plant J. 40: 633-646. 